\title{
Immunosuppressive effects of tick protein RHcyst- 1 on murine bone marrow-derived dendritic cells
}

\author{
Nana Wei ', Zhibing Lin ${ }^{1}$, Zhengmao Xu' ${ }^{1}$, Haiyan Gong ${ }^{1}$, Houshuang Zhang ${ }^{1}$, Yongzhi Zhou ${ }^{1}$, Jie Cao , \\ Guoqing $\mathrm{Li}^{2}$ and Jinlin Zhou ${ }^{1 *}$
}

\begin{abstract}
Background: Ticks, as blood-feeding arthropod vectors, have evolved their own unique mechanism to suppress host immune responses and evade immune defenses in order to complete blood-feeding. The immunoregulatory effect of tick bioactive molecules on hosts has been widely reported, and the cystatin family has been identified as one of the major immunomodulators. In previous studies, we obtained a novel tick salivary bioactive protein named RHcyst-1, which belongs to the type 1 cystatin family. Here, we demonstrated the effects of RHcyst- 1 on the host immune response mainly on dendritic cell (DC) function. Understanding the function of tick-derived bioactive molecule may help to clarify the mechanisms of how ticks escape the host immune response and help to control ticks and tickborne disease transmission.
\end{abstract}

Methods: Bone marrow-derived DCs (BMDCs) were generated and induced by GM-CSF and IL-4 with or without RHcyst-1 addition. Flow cytometry was used to analyze the differentiation and maturation of BMDCs and T cell cytokine production. Quantitative real-time PCR ( $($ RTT-PCR) and western blot were used to measure changes in expression within STAT and p38 MAPK signaling pathways.

Results: Flow cytometry analysis revealed that RHcyst- 1 inhibited the differentiation of BMDCs, but had no effect on the maturation of BMDCs. T cells co-cultured with DCs treated with RHcyst-1 produced significantly less TNF- $a$, IFN- $\gamma$ and IL-2 than the control group. Further analysis showed that the mRNA level and phosphorylation of p38, ERK and STAT were significantly changed after RHcyst-1 added to bone marrow monocytes during the differentiation stage.

Conclusions: Our results suggest that RHcyst-1 is one of the major immunosuppressive proteins of BMDC function from blood-feeding ticks.

Keywords: Tick, RHcyst-1, Cystatin, Dendritic cells, Immune response

\section{Background}

Ticks are obligate hematophagous ectoparasites. During blood-feeding, ticks transmit multiple pathogenic agents which can cause different zoonoses, such as fever with thrombocytopenia syndrome, rickettsiosis, Lyme disease, tick-borne encephalitis, ehrlichiosis, and human granulocytic anaplasmosis [1]. In order to finish the blood meal

\footnotetext{
*Correspondence: jinlinzhou@shvri.ac.cn

${ }^{1}$ Key Laboratory of Animal Parasitology of Ministry of Agriculture, Shanghai Veterinary Research Institute, Chinese Academy of Agricultural Sciences, Shanghai, China

Full list of author information is available at the end of the article
}

and facilitate pathogen transmission, ticks produce many bioactive molecules, which contribute to ticks evading surveillance from the host immune system. These bioactive molecules have anti-hemostatic, anti-inflammatory, anti-complement and immunomodulatory properties. These bioactive molecules contain multiple proteases and protease inhibitors, among which cysteine protease inhibitors such as cystatins represent one of the most prominent protein families $[2,3]$.

Cystatins are reversible and tight-binding inhibitors of cysteine proteases and they are distributed in almost all organisms [4]. Cystatins are divided into three subgroups,

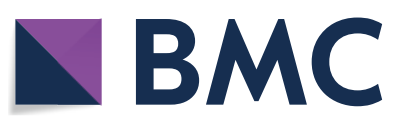

(c) The Author(s) 2019. This article is distributed under the terms of the Creative Commons Attribution 4.0 International License (http://creativecommons.org/licenses/by/4.0/), which permits unrestricted use, distribution, and reproduction in any medium, provided you give appropriate credit to the original author(s) and the source, provide a link to the Creative Commons license, and indicate if changes were made. The Creative Commons Public Domain Dedication waiver (http://creativecommons.org/ publicdomain/zero/1.0/) applies to the data made available in this article, unless otherwise stated. 
including type 1 (stefins), type 2 (cystatins) and type 3 (kininogens) [5]. Cystatins can regulate many biological processes, including antigen processing/presentation, phagocytosis, inflammation, $\mathrm{T}$ cell proliferation and tumor progression $[6,7]$. Cystatin $\mathrm{F}$ is expressed primarily in immune cells and is an important regulator of cell cytotoxicity [8]. Cystatin SA induces IFN- $\gamma$ expression in CD4+ T cells [9]. In human tissues and cells, cystatin $C$ is the most abundant cystatin and may modulate the function of different immune cells [10]. In ticks, to date, only type 1 and type 2 cystatins have been reported, and more studies have been focused on type 2 cystatin in ticks because of their secretory nature [3]. Tick salivary cystatin $\mathrm{OmC} 2$ compromises human DC function by targeting cathepsins S and C [11]. DsCystatin, a novel immunosuppressive protein, inhibits TLR2 and TLR4-directed NFKB activation and also alleviates joint inflammation in mouse models of Borrelia burgdorferi- or complete Freund's adjuvant-induced arthritis [12].

Cystatin RHcyst-1 (GenBank: KM588364), belonging to the type 1 cystatin family, has been detected in embryos, larvae, nymphs and adults, but is most highly expressed at the embryo stage. The RNAi of cystatin RHcyst-1 decreases the rate of embryo hatching. It has been reported that RHcyst-1 has a broad spectrum of inhibitory activities against mammalian cysteine proteases [13]. DCs are the most powerful antigen-presenting cells (APCs) and are key regulators in the immune system. In DCs, mammalian cysteine proteases, especially cathepsins, play important roles in antigen processing and presentation [14]. In this study, we show that RHcyst-1, identified from Rhipicephalus haemaphysaloides, significantly inhibits BMDC differentiation from precursors and impairs the production of cytokines. Hence, RHcyst-1 is an effective immunosuppressive molecule, which helps ticks to evade the host immune response.

\section{Methods}

\section{Experimental animals}

C57BL/6 mice (4-6 weeks of age) were purchased from Slac Laboratory Animal, Inc. (Shanghai, China) and maintained in a SPF facility under sterile conditions at the Animal Center of the Shanghai Veterinary Research Institute (IACUC approve number shvri-mo-2018010019). All animal experiments were performed with permission from local animal-ethics committees.

\section{RNA extraction and $\mathrm{qRT}-\mathrm{PCR}$ experiments}

Total RNA was extracted from different tick organs or cells using TRIzol ${ }^{\circledR}$ (Life Technologies, CA, USA). RNA was reverse transcribed into cDNA using Prime Script $^{\mathrm{TM}}$ RT Reagent with genomic DNA removal (Takara, Dalian, China) by following the manufacturer's
Table 1 Primer sequences used in qRT-PCR analysis. F and R indicate forward and reverse primers, respectively

\begin{tabular}{|c|c|c|}
\hline Gene name & Primer sequence $\left(5^{\prime}-3^{\prime}\right)$ & Length (bp) \\
\hline \multirow[t]{2}{*}{ RHcyst-1 } & F: CACAGTCAGGGAGATTTGCG & \multirow[t]{2}{*}{96} \\
\hline & R:TGCGTGCGATACTTCAGAGG & \\
\hline \multirow[t]{2}{*}{ ELF1a } & F: CGTCTACAAGATTGGTGGCATT & \multirow[t]{2}{*}{108} \\
\hline & R: CTCAGTGGTCAGGTTGGCAG & \\
\hline \multirow[t]{2}{*}{ STAT3 } & F: CACCTTGGATTGAGAGTCAAGAC & \multirow[t]{2}{*}{112} \\
\hline & R: AGGAATCGGCTATATTGCTGGT & \\
\hline \multirow[t]{2}{*}{ STAT4 } & F: GCAGCCAACATGCCTATCCA & \multirow[t]{2}{*}{97} \\
\hline & R:TGGCAGACACTTTGTGTTCCA & \\
\hline \multirow[t]{2}{*}{ STAT5 } & F: CGCCAGATGCAAGTGTTGTAT & \multirow[t]{2}{*}{113} \\
\hline & R: TCCTGGGGATTATCCAAGTCAAT & \\
\hline \multirow[t]{2}{*}{ p38 } & F: CTGACCGACGACCACGTTC & \multirow[t]{2}{*}{118} \\
\hline & R: CTTCGTTCACAGCTAGGTTGC & \\
\hline \multirow[t]{2}{*}{ ERK } & F: CAGGTGTTCGACGTAGGGC & \multirow[t]{2}{*}{139} \\
\hline & R:TCTGGTGCTCAAAAGGACTGA & \\
\hline \multirow[t]{2}{*}{ Mus-actin } & F: AGAGGGAAATCGTGCGTGAC & \multirow[t]{2}{*}{195} \\
\hline & R: CCATACCCAAGAAGGAAGGCT & \\
\hline
\end{tabular}

recommendations. Next, qRT-PCR was performed using $\mathrm{SYBR}^{\circledR}$ Premix Dimmer Eraser kit (Takara) for 40 amplification cycles at $95^{\circ} \mathrm{C}$ for $30 \mathrm{~s}, 95^{\circ} \mathrm{C}$ for $5 \mathrm{~s}$ and $60^{\circ} \mathrm{C}$ for $34 \mathrm{~s}$. The relative expression level of RHcyst-1 in different tick organs was evaluated using the $2^{-\Delta \mathrm{Ct}}$ method. ELF1 $\alpha$ was used as the internal control. ERK, p38, STAT3, STAT4 and STAT5 transcription were evaluated using the $2^{-\Delta \Delta \mathrm{Ct}}$ method [15]. Mouse $\beta$-actin, a housekeeping gene, was used as the internal control. All qRT-PCR amplifications were performed in triplicate with three independent experiments. All qRT-PCR primers used in this study are listed in Table 1.

\section{Immunofluorescence assay (IFA)}

After dissection in PBS, salivary glands were fixed with $4 \%$ paraformaldehyde, dehydrated in a graded series of ethanol and embedded in paraffin. Five micrometer sections were cut and mounted on glass slides. The paraffin was removed from the sections with xylene and the sections were hydrated by successive 5 -min washes with a graded series of 100, 85 and $75 \%$ ethanol and distilled water. Fifteen minutes of antigen retrieval by microwave with ETDA buffer solution (pH 8.0) was performed, followed by washing with PBS three times. The slides were blocked with $3 \%$ BSA for $30 \mathrm{~min}$ at room temperature. Then, the slides were incubated overnight at $4{ }^{\circ} \mathrm{C}$ with primary antibodies and after three washes in PBS, followed for $1 \mathrm{~h}$ with goat-anti-mouse IgG conjugated with FITC (Thermo Fisher, Waltham, MA, USA). The slides were washed with PBS and stained with $10 \mathrm{mg} / \mathrm{ml}$ DAPI for $5 \mathrm{~min}$ at room temperature (Sigma-Aldrich, St. Louis, 
MO, USA). The sections were imaged using a confocal laser-scanning microscope (Zeiss LSM 880, Oberkochen, Germany). The parameters for all the experimental and control groups were set at the same values.

\section{Generation of BMDCs}

BMDCs were generated according to the previously described procedure by Inaba et al. [16], with some modifications. Briefly, bone marrow-derived monocytes from tibias and femurs of C57BL/6 mice were cultured in RPMI-1640 complete medium supplemented with $10 \%$ FBS (Gibco, Grand Island, NY, USA), $100 \mathrm{U} / \mathrm{ml}$ penicillin (Gibco) and $100 \mu \mathrm{g} / \mathrm{ml}$ streptomycin (Gibco). On day $0,5 \times 10^{6}$ cells were seeded into $100-\mathrm{mm}$ dishes (Corning, Corning, NY, USA) in $10 \mathrm{ml}$ RPMI-1640 complete medium plus $40 \mathrm{ng} / \mathrm{ml}$ GM-CSF (Pepro Tech, Rocky Hill, NJ, USA) and $20 \mathrm{ng} / \mathrm{ml} \mathrm{IL-4} \mathrm{(Pepro} \mathrm{Tech).} \mathrm{On} \mathrm{day}$ 3 , another $10 \mathrm{ml}$ of RPMI-1640 complete medium plus $20 \mathrm{ng} / \mathrm{ml} \mathrm{GM}$-CSF was added to the dish. On days 6 and 8 , half of the supernatants were removed and replaced with $10 \mathrm{ml}$ fresh complete medium with sufficient GMCSF and IL-4. On day 9, non-adherent cells were collected and used as immature BMDCs.

To analyze the effects of two cystatins on BMDC differentiation, highly purified RHcyst-1 $(1.8 \mu \mathrm{M})$ was added into the above isolated cells beginning on day 0 of culture and the cells were harvested on day 3 and day 6 and analyzed by flow cytometry (Beckman CytoFLEX, Miami, FL, USA). To analyze the effects of RHcyst-1 on BMDC maturation, the immature BMDCs were activated by the addition of $1 \mu \mathrm{g} / \mathrm{ml}$ LPS (Sigma-Aldrich) with or without RHcyst- 1 treatment. After $18 \mathrm{~h}$ of incubation, the cells were harvested and the expression of CD40, CD80, CD86 and MHC class II molecules was analyzed by flow cytometer.

\section{T cell isolation and co-culture with DCs}

C57BL/6 mice T cells from spleens were isolated according to the Mouse spleen T Cell Isolation Kit's instructions (TBD sciences, Tianjin, China). Differentiated BMDCs with GM-CSF and IL-4, as described above were collected on day 9 and incubated with $1 \mathrm{mg} / \mathrm{ml}$ OVA (GL Biochem, Shanghai, China) with or without RHcyst-1 $(1.8 \mu \mathrm{M})$ treatment for $24 \mathrm{~h}$. DCs were co-cultured with $\mathrm{T}$ cells from spleen in the presence of $1 \mathrm{mg} / \mathrm{ml}$ OVA with or without RHcyst-1 treatment for another $72 \mathrm{~h}$. To determine cytokine production in $\mathrm{DC}$ and $\mathrm{T}$ cell co-cultures by flow cytometry, PMA, Ionomycin (R\&D Systems, Minneapolis, MN, USA) and protein transport inhibitors (BD, Franklin Lakes, NJ, USA) were used. Then, the cells were collected for cytokine analysis by flow cytometry.

\section{Flow cytometry analysis}

For cell-surface staining, cells with different treatments were collected and washed with PBS, and then incubated with FcR-blocking reagent for $20 \mathrm{~min}$ at $4{ }^{\circ} \mathrm{C}$. The cells were then washed and stained by using the following $\mathrm{mAbs}$ (BD): anti-CD11b-FITC, anti-CD11c-APC, anti-CD40-PB450, anti-CD80-PE, anti-CD86-PE, antiMHC-II-PB450, anti-IFN- $\gamma$-APC, anti-TNF- $\alpha$-PB450, anti-IL-2-PE and isotype-matched mAbs for control staining. Finally, the cells were resuspended in $100 \mu \mathrm{l}$ of staining buffer for flow cytometry analysis (Beckman CytoFLEX). Data were analyzed with FlowJo software (Tree Star, Ashland, OR, USA).

\section{Western blot}

Cells were harvested and lysed with IP buffer (Beyotime, Suzhou, China) for $20 \mathrm{~min}$ on ice and centrifuged for $10 \mathrm{~min}\left(12,000 \mathrm{rpm}, 4{ }^{\circ} \mathrm{C}\right)$. Protein concentrations were quantified using a BCA protein assay kit (Thermo Fisher). Then, $20-50 \mu \mathrm{g}$ total proteins from each sample were electrophoresed in a $12 \%$ SDS-AGE gel and transferred onto polyvinylidene fluoride (PVDF) membranes (Millipore, Billerica, MA, USA). The membranes were blocked with $5 \%$ nonfat milk for $1 \mathrm{~h}$ at $37{ }^{\circ} \mathrm{C}$ and probed with anti-phosphorylated or unphosphorylated p38 MAPK, p44/42 MAPK (Erk1/2), STAT3 and STAT5 antibody (CST, Danvers, MA, USA) overnight at $4{ }^{\circ} \mathrm{C}$. The bone marrow-derived monocyte induced with GM-CSF/ IL-4 stimulation without RHcyst-1 treatment was used as negative control. The membranes were washed with TBST and then probed with secondary antibody goat anti-mouse or goat anti-rabbit IgG (Thermo Fisher) for $1 \mathrm{~h}$ at $37^{\circ} \mathrm{C}$. Band signals were visualized with Pierce ${ }^{\mathrm{TM}}$ ECL Western Blotting Substrate (Thermo Fisher) under a Multiple-color Fluorescence Imaging System (Bio-Rad, Hercules, CA, USA).

\section{Statistical analysis}

All statistical analyses were performed using GraphPad Prism 6 software (GraphPad Software Inc., La Jolla, CA, USA). Numerical data are presented as the mean $\pm S D$, and most of the data were analyzed by Student's t-test. In all statistical analyses, * indicates $P<0.05$, ** indicates $P<0.01$, ${ }^{* * * *}$ indicates $P<0.001$ and NS indicates no significance.

\section{Results \\ Expression analysis of RHcyst-1}

To determine the expression profile of RHcyst-1, total RNA from adult ticks' salivary gland (SG), midgut (MD), fat body (FB), ovary (OV) were subjected to qRTPCR testing. The primers used in this study are shown 

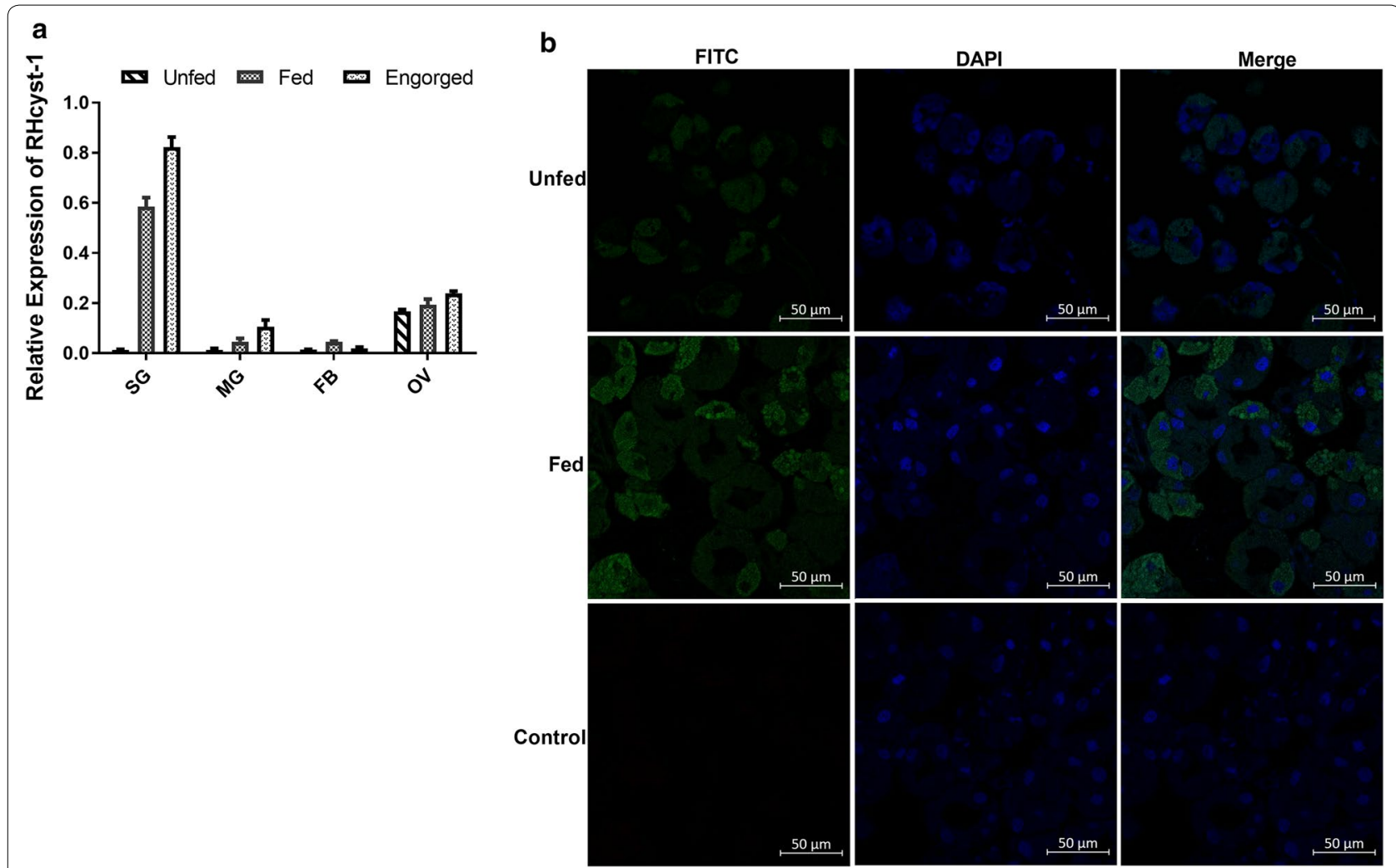

Fig. 1 The expression and distribution of RHcyst-1. a Gene expression patterns of RHcyst-1, isolated from Rhipicephalus haemaphysaloides, in different organs from unfed, fed and engorged adult ticks using qRT-PCR. b Distribution of native RHcyst-1 in SG from unfed and fed adult ticks. Bars represent the mean $\pm S D$. Data are representative of three independently repeated experiments. Abbreviations: SG, salivary glands; $M D$, midgut; FB, fat body; OV, ovary

in Table 1. As shown in Fig. 1a, RHcyst-1 was highly expressed in the SG, and the expression in the SG was upregulated from unfed to engorged ticks. Furthermore, we investigated the distribution of RHcyst-1 in the SG of unfed and fed ticks using IFA. We found that RHcyst-1 was highly expressed in the SG during blood-feeding (Fig. 1b). These results suggest that RHcyst-1 is a salivary gland protein and may play an important role during tick blood-feeding.

\section{RHcyst-1 impairs differentiation of BMDC}

Tick salivary gland proteins involved in host immune responses have been widely reported. To explore the effect of RHcyst-1 on host immune responses, we focused on DC function. DCs are pivotal APCs, which bridge innate and adaptive immune systems and are the initiator and modulator of the immune response [17]. To search for the effect of RHcyst-1 on the differentiation of DCs, bone marrow-derived monocytes were induced with GM-CSF/IL-4 in the presence or absence of RHcyst-1, respectively. In order to verify whether the effect was directed against an early or a late phase of BMDC differentiation, the cells were collected on the third and sixth day and the ratio of BMDCs was evaluated by flow cytometry. As shown in Fig. 2, RHcyst-1 significantly inhibited the differentiation of bone marrow monocytes into CD11c+ CD11b+ BMDCs, whether it was on day 3 (RHcyst-1: $8.16 \pm 0.70 \%$ vs control: $21.44 \pm 0.50 \%$; t-test: $\left.t_{(4)}=15.4, P=0.0001\right)$ or day 6 (RHcyst-1: $27.37 \pm 0.43 \%$ vs control: $\left.64.97 \pm 1.28 \% ; t_{(4)}=27.75, P=0.00001\right)$.

In addition, the safety of RHcyst- 1 on the normal cell lines 293T, Vero and $\mathrm{CHO}$ has been evaluated in our previous studies. RHcyst- 1 treatment did not change the activity of normal cells and did not induce apoptosis in these cell lines [18]. These results show that the suppression of BMDC differentiation by RHcyst- 1 is not due to apoptosis or cytotoxicity.

\section{RHcyst-1 shows no effect on BMDC maturation}

In the following experiments, we analyzed the effect of RHcyst-1 on LPS- stimulated maturation of BMDCs. Bone marrow monocytes were cultured in the presence of GM-CSF and IL-4 for 9 days, and the differentiated immature DCs were harvested. The harvested 

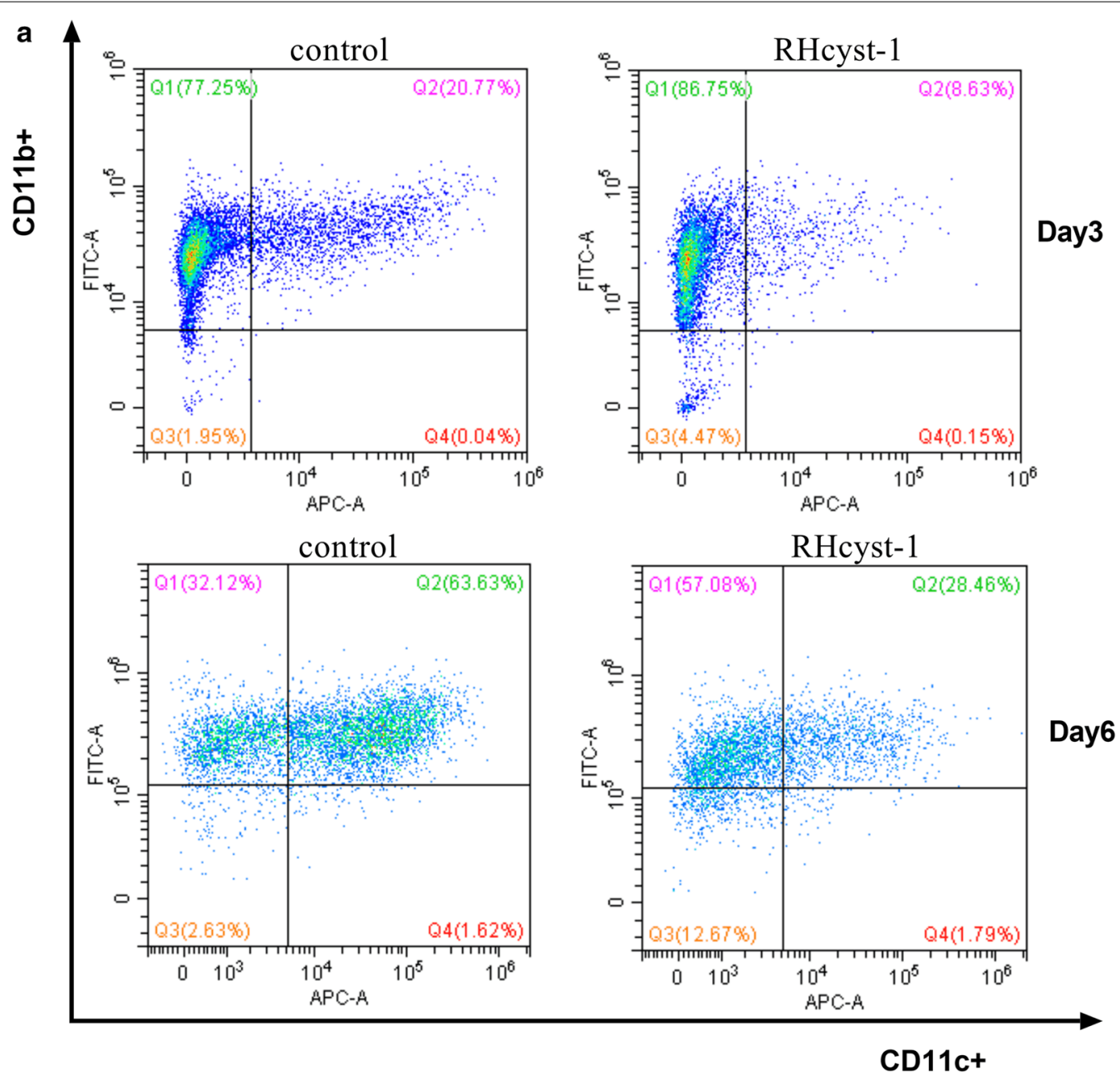

b

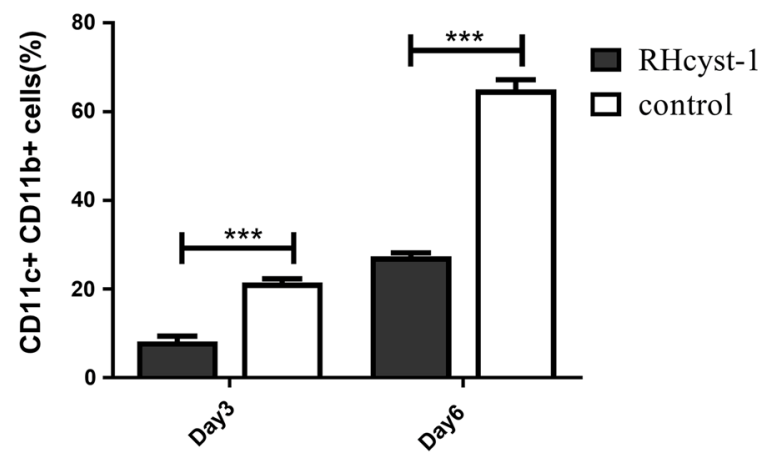

Fig. 2 RHcyst-1 modulates the differentiation of bone marrow-derived monocytes into DCs. Bone marrow monocytes were cultured in GM-CSF and IL-4 condition (control) or in the presence of RHcyst-1. CD11 C+ CD11b+ cells were analyzed by flow cytometry. a Differentiated cells (CD11c+ $\mathrm{CD} 11 \mathrm{~b}+$ ) are shown in dot plots. $\mathbf{b}$ The representative of three independent experiments is shown. ${ }^{* * *}$ indicates $P<0.001$; bars represent the mean \pm SD

BMDCs were stimulated by LPS for $18 \mathrm{~h}$ with or without RHcyst-1. Then, the BMDCs were stained and analyzed for the expression of CD40, CD80, CD86 and MHC-II molecules by flow cytometry. The immature DCs stimulated with LPS showed significantly increased expression of CD40 (one-way ANOVA: $F_{(2,6)}=3477, P=0.00001$ ) 


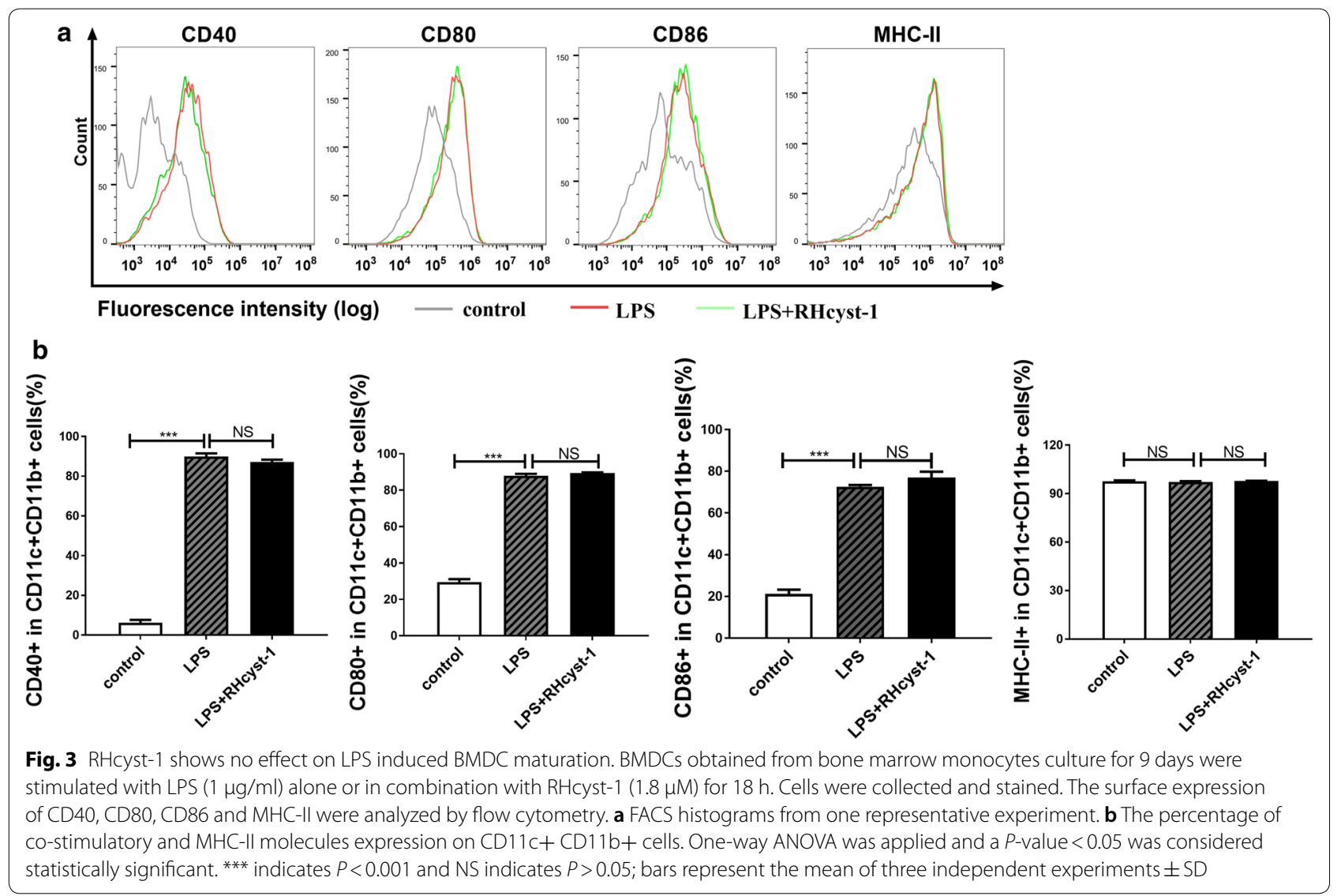

$\operatorname{CD} 80\left(F_{(2,6)}=2477, P=0.00001\right)$ and $\operatorname{CD} 86\left(F_{(2,6)}=697.5\right.$, $P=0.00001)$ compared with that of the control group. However, there was no change on LPS-induced upregulation of CD40 (one-way ANOVA: $F_{(2,6)}=3477, P=0.0953$ ), CD80 $\left(F_{(2,6)}=2477, P=0.3282\right), C D 86 \quad\left(F_{(2,6)}=697.5\right.$, $P=0.0638)$ and MHC-II $\left(F_{(2,6)}=1.805, P=0.1841\right)$ when BMDCs were exposed to RHcyst-1 (Fig. 3). These results indicate that RHcyst-1 has no effect on DC maturation.

\section{RHcyst-1 inhibits cytokine levels in BMDC}

The levels of cytokines are critical in the regulation of $\mathrm{DC}$ function because of their ability to activate $\mathrm{T}$ cell responses. Thus, we analyzed cytokine levels in RHcyst1-treated BMDCs in response to OVA stimulation. As shown in Fig. 4a, b, a significant reduction was detectable following RHcyst-1 treatment for IL-2, TNF- $\alpha$ and IFN- $\gamma$, after OVA-CD4 (t-test: $t_{(4)}=7.918, P=0.002 ; t_{(4)}=7.665$, $\left.P=0.0016 ; t_{(4)}=7.539, P=0.0017\right)$ and OVA-CD8 (t-test: $t_{(4)}=5.563, P=0.0051 ; t_{(4)}=5.056, P=0.0072 ; t_{(4)}=6.257$, $P=0.0033)$ peptide stimulated, respectively. In conclusion, RHcyst-1 inhibits the level of cytokines which are important for $\mathrm{T}$ cell activation.

\section{Cell signaling pathways induced by RHcyst-1 during BMDC} differentiation

The p38 MAPK signaling pathway is crucial for the differentiation of BMDC [14]. Combined inhibition of STAT3 and p38 MAPK could prevent tumor-mediated suppression of monocyte-derived DC differentiation [19]. To examine signaling pathways of RHcyst-1 involved in bone marrow monocytes differentiated into DCs, cells from day 6 cultures were prepared for western blot analyses. As shown in Fig. 5a, the mRNA levels of p38 (t-test: $\left.t_{(2)}=13.92, P=0.0051\right)$, ERK $\left(t_{(2)}=8.964, P=0.0122\right)$, STAT3 $\left(t_{(2)}=6.01, P=0.0266\right)$ and STAT5 $\left(t_{(2)}=10.44\right.$, $P=0.0091)$ were all upregulated compared to those of the control group. The primer sets used in this study are shown in Table 1. We also tested the phosphorylation levels of p38 MAPK and STAT signaling pathways. As shown in Fig. 5b, c, decreased pp38 (t-test: $t_{(4)}=5.061$, $P=0.0072)$ and pERK $\left(t_{(4)}=11.96, P=0.0003\right)$ levels and increased pSTAT3 $\left(t_{(4)}=5.53, P=0.0052\right)$ and pSTAT5 $\left(t_{(4)}=14.25, P=0.0001\right)$ levels were observed in cells treated with RHcyst-1 compared with those in the control group. These results show that RHcyst- 1 inhibits the p38 MAPK pathway and activates the STAT signaling pathway during the differentiation of BMDC. 

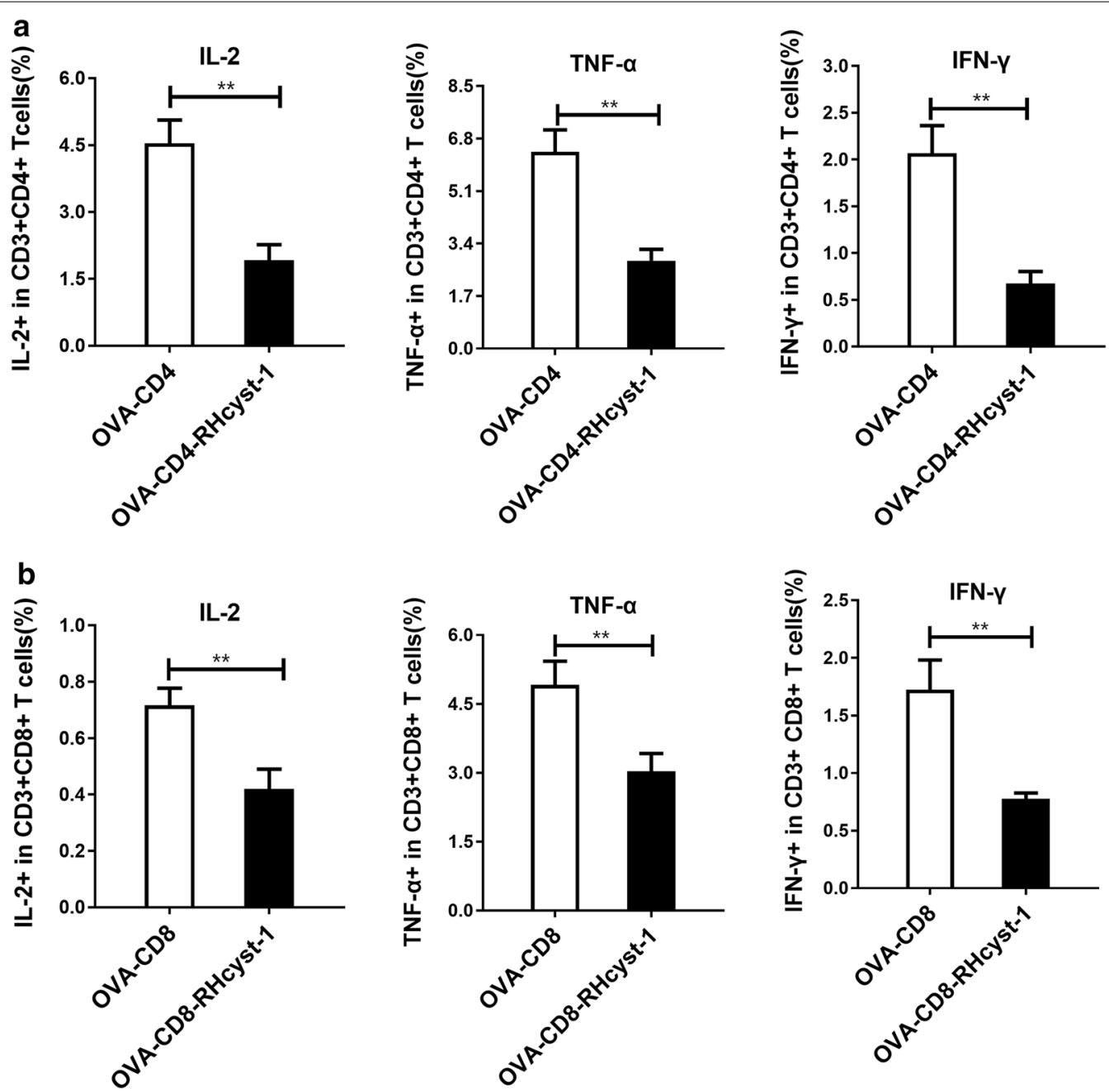

Fig. 4 RHcyst-1 inhibits T cell activation and cytokine production. BMDCs were stimulated with OVA-CD4 (a) and OVA-CD8 (b) peptide with or without RHcyst-1 treatment, respectively. The above BMDCs were co-cultured with T cells from spleen for another $72 \mathrm{~h}$. The levels of IL-2, TNF-a and IFN- $\gamma$ were measured by flow cytometry. Student's t-test was applied and a $P$-value $<0.05$ was considered statistically significant. ${ }^{* *}$ indicates $P<0.01$; bars represent the mean of three independent experiments $\pm S D$

\section{Discussion}

Immunosuppression that occurs during the process of tick blood-feeding has been reported extensively [2023]. Saliva and salivary gland extracts are involved in the modulation of host immunity [24]. Tick salivary gland extracts are composed of multiple proteins belonging to different protein families, such as the cystatin family, serpin family and Kunitz inhibitor family. In this study, we found that RHcyst-1 is highly expressed in salivary glands and upregulated during tick blood-feeding (Fig. 1a, b). Our results demonstrate the immune inhibitory properties mainly on DC function of a salivary gland cystatin, RHcyst-1, isolated from the tick Rhipicephalus haemaphysaloides.
DCs are powerful antigen-presentation cells and are essential in initiating adaptive immune responses in hosts [17]. DCs can also directly contact and affect pathogen infection and be targeted by ticks to suppress the initiation of adaptive immune responses in hosts $[25,26]$. In this study, we demonstrate that RHcyst-1 significantly inhibits the differentiation of BMDC and suppresses DC-induced T-lymphocyte activation. There have been some previous reports about tick cystatins on DC function [2, 27, 28]. However, how tick cystatin regulates the host immune signaling pathway and impairs the host immune cell function remains largely unclear.

Many tick cystatins belonging to type 1 and 2 families have been reported. Studies of the function of tick 

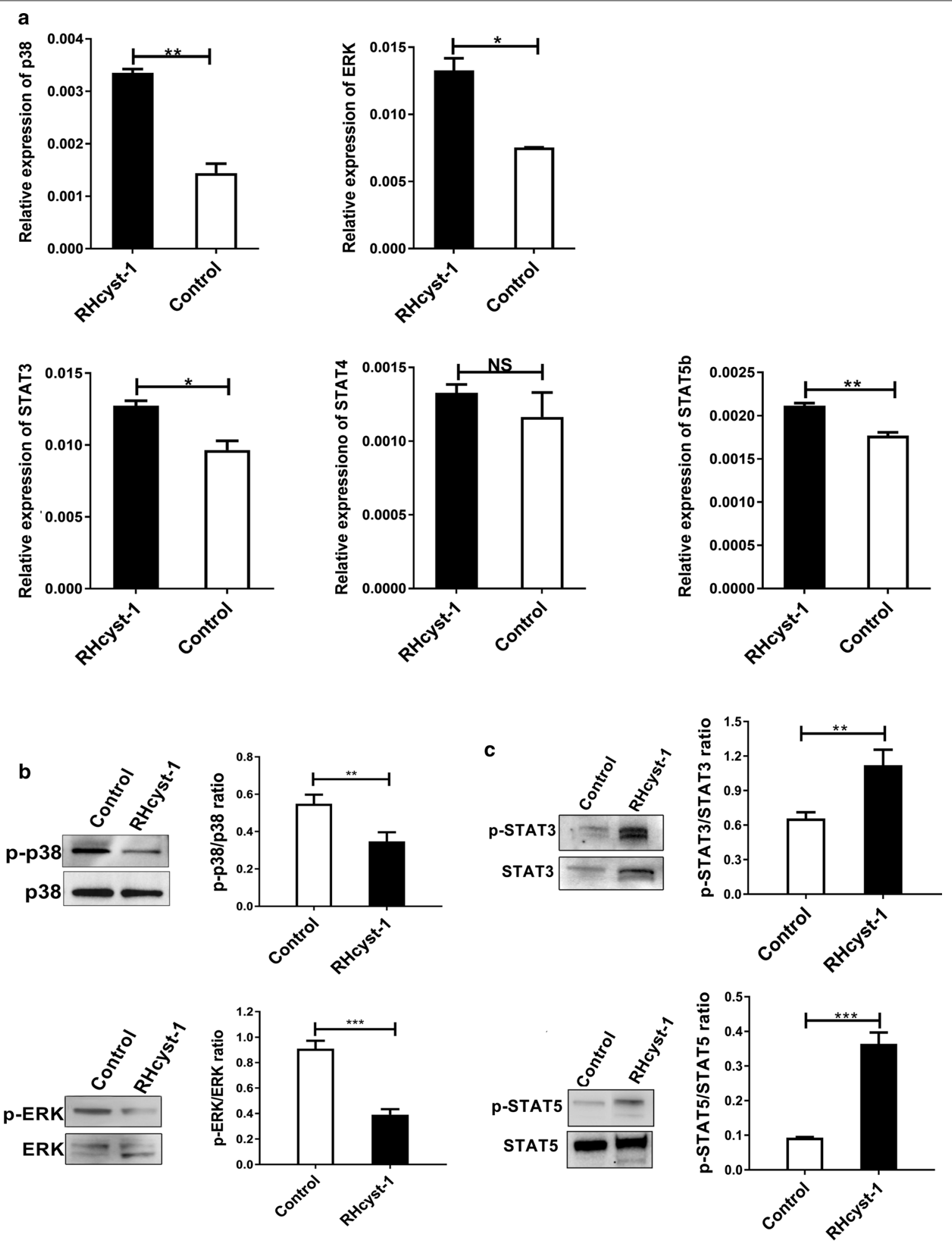

Fig. 5 RHcyst-1 affects p38 MAPK and STAT signaling pathways during the differentiation BMDCs. a The mRNA expression of p38 MAPK and STAT signaling pathways during BMDC differentiation after RHcyst-1 treatment was quantified by qRT-PCR. The effect of RHcyst-1 on phosphorylation of p38 MAPK pathway (b) and STAT pathway (c) during BMDCs differentiation was determined by western blot. Student's t-test was applied and a $P$-value $<0.05$ was considered statistically significant. ${ }^{*} P<0.05,{ }^{* *} P<0.01,{ }^{* * *} P<0.001$ and NS indicates $P>0.05$; bars represent the mean \pm SD 
cystatins in the host have primarily focused on the type 2 family [2]. In our previous study, we reported that RHcyst-1 belongs to the type1 cystatin family and is involved in tick physiology [13]. In this study, although RHcyst-1 was not directly detectable in serum from rabbits on which ticks had repeatedly fed (Additional file 1: Figure S1), previous studies have shown that RHcyst-1 has a broad spectrum of mammalian cathepsin-inhibition activities and mediates specific immune responses in tumor-bearing mice [13, 18]. Recently, exosomes have been most extensively studied and tickderived exosomes were reported to mediate transmission of tick-borne disease [29-32]. Whether RHcyst-1, as the component of exosomes, enters into the host to regulate the host immune response remains to be further investigated.

Protein phosphorylation is a modification of proteins and is the major molecular mechanism of protein regulation. The STAT and p38 MAPK pathways are tightly associated with DC differentiation [14, 19]. In the present study, we found that RHcyst-1, a cysteine protease inhibitor, inhibited the phosphorylation of p38 and ERK, but increased the phosphorylation of STAT3 and STAT5 during BMDC differentiation. It has been reported that cystatin from the murine nematode parasite, Heligmosomoides polygyrus, alters the $\mathrm{T}$ cell activating functionality of DCs [33]. In this study, in addition to changes in STAT and p38 MAPK signaling pathways, we observed that RHcyst- 1 treatment suppressed $\mathrm{T}$ cell activation by BMDCs. The observations made in our study may represent one of the important mechanisms by which blood-feeding ticks induce immunosuppression in their hosts. Regulating DC differentiation and function may be a potential strategy for ticks to promote blood-feeding and pathogen transmission. More effort is being done to study the critical roles of biologically important tick bioactive molecules in tick-pathogen-hosts [34-36]. RHcyst- 1 may be a promising target for tick control in the future.

\section{Conclusions}

We show here that the tick type 1 cystatin, RHcyst-1, affects different functions of BMDCs. In particular, RHcyst-1 significantly inhibits BMDC differentiation, in part through p38 MAPK inhibition and STAT activation. Our results contribute to clarifying tick-host interactions and provide a better understanding of the mechanisms used by tick bioactive molecules to escape host immune system detection and allow ticks to successfully feed.

\section{Additional file}

Additional file 1: Figure S1. Western blot analysis of RHcyst-1. Total 20 $\mu \mathrm{g}$ of purified RHcyst-1 was electrophoresed in SDS-PAGE gel and then transferred onto PVDF membrane for Western blot analysis with serum from rabbits on which ticks had repeatedly fed (a) and normal rabbit serum as control (b).

\section{Abbreviations}

DC: dendritic cell; BMDC: bone marrow-derived DC; APCs: antigen-presenting cells; ERK: extracellular-regulated protein kinases; MAPK: mitogen-activated protein kinase; STAT: signal transducers and activators of transcription; cystatin: cysteine protease inhibitor; ELF1a: elongation factor-1 gene; PBS: phosphatebuffered saline; OVA: ovalbumin; TNF-a: tumor necrosis factor-alpha; IFN-y: interferon-gamma; L-2: interleukin-2; IL-4: interleukin-4; GM-CSF: granulocytemacrophage colony stimulating factor.

\section{Acknowledgements}

We thank the support and help of our colleagues Yanan Wang and Jinmiao Lu. We thank LetPub (http://www.letpub.com) for its linguistic assistance during the preparation of this manuscript.

\section{Funding}

This work was supported by the "National Key Research and Development Program of China" (2017YFD0501200) and the "National Natural Science Foundation of China" (31711540022) and the "China Postdoctoral Science Foundation" (2018M641567).

\section{Availability of data and materials}

The data supporting the conclusions of this article are included within the article and its additional file.

\section{Authors' contributions}

NW designed the projects, collected and analyzed the data, and wrote the manuscript. NW, ZL and ZX carried out all the experiments and collected the data. NW and JZ designed the study, and reviewed and revised the manuscript. HZ, HG, YZ and JC helped in the mouse-housed experiments. All authors read and approved the final manuscript.

\section{Ethics approval and consent to participate}

Mice were maintained in a SPF facility under sterile conditions at the Animal Center of the Shanghai Veterinary Research Institute (IACUC approve number shvri-mo-2018010019). All animal experiments were authorized by the Animal Ethical Committee of Shanghai Veterinary Research Institute.

\section{Consent for publication}

Not applicable.

\section{Competing interests}

The authors declare that they have no competing interests.

\section{Publisher's Note}

Springer Nature remains neutral with regard to jurisdictional claims in published maps and institutional affiliations.

\section{Author details}

${ }^{1}$ Key Laboratory of Animal Parasitology of Ministry of Agriculture, Shanghai Veterinary Research Institute, Chinese Academy of Agricultural Sciences, Shanghai, China. ${ }^{2}$ Guangdong Provincial Zoonosis Prevention and Control Key Laboratory, College of Veterinary Medicine, South China Agricultural University, Guangzhou, China.

Received: 2 November 2018 Accepted: 26 March 2019

Published online: 15 April 2019 


\section{References}

1. Hajdusek O, Sima R, Ayllon N, Jalovecka M, Perner J, de la Fuente J, et al. Interaction of the tick immune system with transmitted pathogens. Front Cell Infect Microbiol. 2013;3:26.

2. Chmelar J, Kotal J, Langhansova H, Kotsyfakis M. Protease inhibitors in tick saliva: the role of serpins and cystatins in tick-host-pathogen interaction. Front Cell Infect Microbiol. 2017:7:216.

3. Schwarz A, Valdes JJ, Kotsyfakis M. The role of cystatins in tick physiology and blood feeding. Ticks Tick Borne Dis. 2012;3:117-27.

4. Kordis D, Turk V. Phylogenomic analysis of the cystatin superfamily in eukaryotes and prokaryotes. BMC Evol Biol. 2009;9:266.

5. Turk V, Stoka V, Turk D. Cystatins: biochemical and structural properties, and medical relevance. Front Biosci. 2008;13:5406-20.

6. Zhang J, Shridhar R, Dai Q, Song J, Barlow SC, Yin L, et al. Cystatin m: a novel candidate tumor suppressor gene for breast cancer. Cancer Res. 2004;64:6957-64.

7. Zavasnik-Bergant T. Cystatin protease inhibitors and immune functions. Front Biosci. 2008:13:4625-37.

8. Prunk M, Perišić Nanut M, Sabotič J, Kos J. Cystatins, cysteine peptidase inhibitors, as regulators of immune cell cytotoxicity. Period Biol. 2017;118:353-62.

9. Kato T, Ito T, Imatani T, Minaguchi K, Saitoh E, Okuda K. Cystatin SA, a cysteine proteinase inhibitor, induces interferon-gamma expression in CD4-positive T cells. Biol Chem. 2004;385:419-22.

10. Zi M, Xu Y. Involvement of cystatin C in immunity and apoptosis. Immunol Lett. 2018;196:80-90.

11. Zavasnik-Bergant T, Vidmar R, Sekirnik A, Fonovic M, Salat J, Grunclova L, et al. Salivary tick cystatin OmC2 targets lysosomal cathepsins $S$ and $C$ in human dendritic cells. Front Cell Infect Microbiol. 2017;7:288.

12. Sun T, Wang F, Pan W, Wu Q, Wang J, Dai J. An immunosuppressive tick salivary gland protein DsCystatin interferes with toll-like receptor signaling by downregulating TRAF6. Front Immunol. 2018;9:1245.

13. Wang $Y$, Zhou $Y$, Gong $H$, Cao J, Zhang H, Li X, et al. Functional characterization of a cystatin from the tick Rhipicephalus haemaphysaloides. Parasit Vectors. 2015:8:140.

14. Xie J, Qian J, Yang J, Wang S, Freeman ME 3rd, Yi Q. Critical roles of Raf/ MEK/ERK and PI3 K/AKT signaling and inactivation of p38 MAP kinase in the differentiation and survival of monocyte-derived immature dendritic cells. Exp Hematol. 2005;33:564-72.

15. Livak KJ, Schmittgen TD. Analysis of relative gene expression data using real-time quantitative PCR and the 2(-Delta Delta $C(T))$ method. Methods. 2001:25:402-8.

16. Inaba K, Inaba M, Romani N, Aya H, Deguchi M, Ikehara S, et al. Generation of large numbers of dendritic cells from mouse bone marrow cultures supplemented with granulocyte/macrophage colony-stimulating factor. J Exp Med. 1992;176:1693-702.

17. Banchereau J, Steinman RM. Dendritic cells and the control of immunity. Nature. 1998;392:245-52.

18. Wei N, Lin Z, Xu Z, Cao J, Zhou Y, Zhang H, et al. A tick cysteine protease inhibitor RHcyst-1 exhibits antitumor potential. Cell Physiol Biochem. 2018:46:2385-400.

19. Oosterhoff $D$, Lougheed S, van de Ven R, Lindenberg J, van Cruijsen $H$, Hiddingh $\mathrm{L}$, et al. Tumor-mediated inhibition of human dendritic cell differentiation and function is consistently counteracted by combined p38 MAPK and STAT3 inhibition. Oncoimmunology. 2012;1:649-58.

20. Ferreira BR, Szabo MJ, Cavassani KA, Bechara GH, Silva JS. Antigens from Rhipicephalus sanguineus ticks elicit potent cell-mediated immune responses in resistant but not in susceptible animals. Vet Parasitol. 2003;115:35-48.

21. Mudenda L, Pierle SA, Turse JE, Scoles GA, Purvine SO, Nicora CD, et al. Proteomics informed by transcriptomics identifies novel secreted proteins in Dermacentor andersoni saliva. Int J Parasitol. 2014:44:1029-37.

22. Francischetti IM, Sa-Nunes A, Mans BJ, Santos IM, Ribeiro JM. The role of saliva in tick feeding. Front Biosci. 2009;14:2051-88.

23. Hovius JW. Spitting image: tick saliva assists the causative agent of Lyme disease in evading host skin's innate immune response. J Invest Dermatol. 2009:129:2337-9.

24. Kotal J, Langhansova H, Lieskovska J, Andersen JF, Francischetti IM, Chavakis T, et al. Modulation of host immunity by tick saliva. J Proteom. 2015;128:58-68.

25. Rescigno M, Urbano M, Valzasina B, Francolini M, Rotta G, Bonasio R, et al. Dendritic cells express tight junction proteins and penetrate gut epithelial monolayers to sample bacteria. Nat Immunol. 2001:2:361-7.

26. Hovius JW, de Jong MA, den Dunnen J, Litjens M, Fikrig E, van der Poll T, et al. Salp15 binding to DC-SIGN inhibits cytokine expression by impairing both nucleosome remodeling and mRNA stabilization. PLoS Pathog. 2008;4:e31.

27. Salat J, Paesen GC, Rezacova P, Kotsyfakis M, Kovarova Z, Sanda M, et al. Crystal structure and functional characterization of an immunomodulatory salivary cystatin from the soft tick Ornithodoros moubata. Biochem J. 2010;429:103-12.

28. Lieskovska J, Palenikova J, Langhansova H, Chagas AC, Calvo E, Kotsyfakis $M$, Kopecky J. Tick sialostatins $L$ and $L 2$ differentially influence dendritic cell responses to Borrelia spirochetes. Parasit Vectors. 2015:8:275.

29. Hackenberg M, Kotsyfakis M. Exosome-mediated pathogen transmission by arthropod vectors. Trends Parasitol. 2018;34:549-52.

30. Zhang X, Yuan X, Shi H, Wu L, Qian H, Xu W. Exosomes in cancer: small particle, big player. J Hematol Oncol. 2015;8:83.

31. Zhou W, Woodson M, Neupane B, Bai F, Sherman MB, Choi KH, et al. Exosomes serve as novel modes of tick-borne flavivirus transmission from arthropod to human cells and facilitates dissemination of viral RNA and proteins to the vertebrate neuronal cells. PLOS Pathog. 2018;14:e1006764.

32. Shkarina KA, Cherednyk OV, Voloschenko II, Trembach OM, Trembach IO, Khoruzhenko Al. Exosomes: messengers and mediators of tumor-stromal interactions. Biopolym Cell. 2014:30:426-35.

33. Sun Y, Liu G, Li Z, Chen Y, Liu Y, Liu B, et al. Modulation of dendritic cell function and immune response by cysteine protease inhibitor from murine nematode parasite Heligmosomoides polygyrus. Immunology. 2013;138:370-81.

34. Hovius JW, van Dam AP, Fikrig E. Tick-host-pathogen interactions in Lyme borreliosis. Trends Parasitol. 2007;23:434-8.

35. Kim TK, Tirloni L, Pinto AF, Moresco J, Yates JR 3rd, da Silva Vaz I, Jr Mulenga A. Ixodes scapularis tick saliva proteins sequentially secreted every 24 h during blood feeding. PLoS Negl Trop Dis. 2016;10:e0004323.

36. Murfin KE, Fikrig E. Tick bioactive molecules as novel therapeutics: beyond vaccine targets. Front Cell Infect Microbiol. 2017;7:222.

Ready to submit your research? Choose BMC and benefit from

- fast, convenient online submission

- thorough peer review by experienced researchers in your field

- rapid publication on acceptance

- support for research data, including large and complex data types

- gold Open Access which fosters wider collaboration and increased citations

- maximum visibility for your research: over 100M website views per year

At BMC, research is always in progress.

Learn more biomedcentral.com/submissions 